\title{
Aus dem Vorwort zur dritten Auflage
}

Die erste Auflage dieses Kommentars wurde im Jahre 1906 von dem damaligen Hamburger Amtsrichter Dr. Georg Schaps herausgegeben. Schaps ist im Sommer 1918 als Reichsgerichtsrat verstorben. So konnte der erste Band der zweiten Auflage nur noch teilweise von ihm vorbereitet werden. Er wurde von dem Präsidenten des Hanseatischen Oberlandesgerichts, Prof. Dr. Max Mittelstein, und dem Königsberger Rechtsanwalt Dr. Julius Sebba zu Ende geführt und erschien im Jahre 1921. Herrn Dr. Sebba lag nach dem Ableben von Herrn Präsidenten Dr. Mittelstein die Bearbeitung des zweiten Bandes der zweiten Auflage, der im Jahre 1929 herausgebracht wurde, allein ob.

Kernstück der beiden ersten Auflagen war die eingehende Erläuterung des vierten Buches des HGB. Um sie herum rankte sich eine Zusammenstellung der übrigen seerechtlichen Bestimmungen, deren Kommentierung nicht in allen Fällen angebracht war. Seither hat sich die Zahl dieser Nebengesetze und -verordnungen nicht nur erheblich vermehrt. Es kommen heute auch einige von ihnen an Bedeutung dem vierten Buch des HGB gleich, so daß sie wie dieses einer eingehenden Erläuterung bedurften. Auch das Seevölkerrecht erforderte gegenüber den früheren Auflagen eine stärkere Beachtung. Ich entschloß mich deshalb, den Kommentar und die Zusammenstellung der Nebenbestimmungen in sechs Teile zu gliedern, in denen die Allgemeine Einleitung, die völkerrechtliche Lage der See, die Rechtslage des Schiffes, das vierte Buch des HGB, das Seearbeitsrecht und die verkehrstechnischen Bestimmungen behandelt sind. Den ersten Band bilden die drei ersten Teile, der zweite ist ausschließlich dem vierten Buch des HGB gewidmet, im dritten sind das Seearbeitsrecht und die verkehrstechnischen Bestimmungen enthalten.

Der Kommentar umfaßt somit grundsätzlich das gesamte Seerecht. Doch finden sich auf das Seekriegsrecht nur gelegentliche Hinweise. Auch ist auf eine Erläuterung der seeversicherungsrechtlichen Bestimmungen bewußt verzichtet worden.

Frankfurt/Main, den 20. Mai 1959

Hans Jürgen Abraham 